Forum 2016 · 31:360

DOI 10.1007/s12312-016-0143-4

Online publiziert: 30. September 2016

๑) Springer-Verlag Berlin Heidelberg 2016

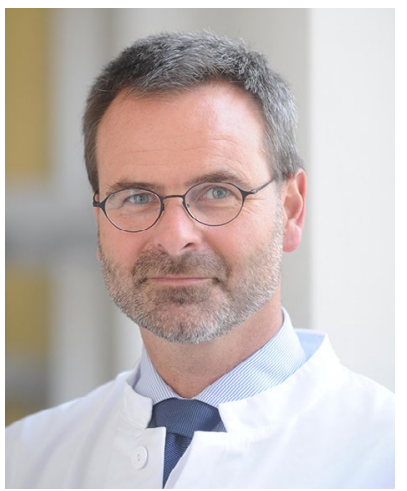

Was genau wollen wir herausfinden? Diese Frage steht am Anfang einer jeden klinischen Studie - die Antwort darauf entscheidet über das geeignete Studiendesign, über Größe, Studiendauer, Studienendpunkte und Messmethoden. Mangelnde Sorgfalt bei der Auswahl dieser Parameter können spürbare Folgen für die Gesundheitsversorgung haben. Das zeigte sich erst kürzlich am Beispiel der amerikanischen PLCO-Studie aus dem Jahr 2009. In dieser Studie ging es, ebenso wie in der europäischen ERSPC-Studie, um die Wirksamkeit des PSA-Screenings bei der ProstatakrebsFrüherkennung. Im Gegensatz zur ERSPCStudie konnte die PLCO-Studie aber keinen Überlebensvorteil für das PSA-Screening nachweisen. 2011 zog die US Preventive Services Task Force (USPSTF) deshalb die Empfehlung für das PSA-Screening in den USA zurück. In einzelnen US-Bundesstaaten sank daraufhin die Inzidenz beim Prostatakarzinom um aktuell bis zu $30 \%$; das betrifft auch die Inzidenz potenziell aggressiver Tumoren. Eine Re-Analyse der Daten ergab jetzt allerdings Fehler im Bericht der PLCO-Ergebnisse, sodass die USPSTF ihre Empfehlung derzeit ausgesetzt hat.

Was war geschehen? Bei der PLCO-Studie handelt es sich um eine randomisiert

Peter Albers

Präsident der Deutschen Krebsgesellschaft e.V.

\title{
Klinische Studien: Ein niedriges Qualitätsniveau können wir uns nicht leisten
}

kontrollierte Studie mit mehr als 76.000 Männern, die in zwei Gruppen eingeteilt wurden - die eine unterzog sich regelmäßig einem PSA-Test, die Kontrollgruppe angeblich nicht. Der Vergleich des Sterberisikos in beiden Gruppen nach sieben Jahren ergab praktisch keinen Unterschied ( $N$ Engl J Med. 2009;360:1310-9). Doch offenbar wurde die Kontamination durch PSATests im Kontrollarm der Studie in der ursprünglichen Auswertung stark unterschätzt. Man war davon ausgegangen, dass sich die Männer im Kontrollarm an die Empfehlung halten würden, keinen PSATest durchführen zu lassen. Doch 9 von 10 Männern hatten den Test trotzdem in Anspruch genommen. Kumulativ kamen im Kontrollarm sogar mehr PSA-Tests zusammen als im Interventionsarm. Kein Wunder, dass kein Unterschied zwischen beiden Studienarmen festgestellt wurde.

Es bleibt die Frage, warum diese hohen Kontaminationsraten erst jetzt auffielen. Interessanterweise hatten die PLCOStatistiker bereits 2010 auf das Problem hingewiesen (Clin Trials. 2010;7:303-11), wenn auch das genaue Ausmaß zu diesem Zeitpunkt nicht klar war. Warum die Verantwortlichen diesem Hinweis nicht weiter nachgingen und die USPSTF damals nicht reagierte, ist schwer zu verstehen.

Solche Probleme sind nicht neu - ich erinnere an die Bezwoda-Studie, die 1995 die Vorteile der Hochdosistherapie beim Mammakarzinom beweisen wollte und deren Publikation 2001 nach einer Recherche aufgrund gefälschter Daten zurückgezogen werden musste (J Clin Oncol. 2001;19(11):2973).

Doch noch immer herrscht ein gravierender Mangel an Transparenz in unserem Wissenschaftssystem. John P. Ioannidis von der Stanford-Universität, Arzt und bekannter
Kritiker des heutigen Forschungsbetriebs, beklagt unter anderem, dass hochwertige Untersuchungen in der Hälfte der Fälle nicht zur Überprüfung zur Verfügung stehen - sie werden oft nicht publiziert, z.B. weil die Verantwortlichen dann Negativergebnisse hätten berichten müssen. Was veröffentlich wird, erscheint meist in lizenzpflichtigen Zeitschriften und ist folglich nur eingeschränkt zugänglich. Studienergebnisse sind oft so verkürzt dargestellt, dass das Reproduzieren schwerfällt. Selten nimmt jemand Einsicht in die Rohdaten; für die medizinischen Leitlinien zählt letztlich, was publiziert wurde. Vor allem: Ein großer Teil der medizinischen Forschungsbemühungen lässt den Patientennutzen außer Acht. Stattdessen wird untersucht, was rasch Veröffentlichungen oder weitere Forschungsmittel einbringt. In Zeiten, in denen die Ergebnisse großer Medikamentenstudien ganze Unternehmensstrategien bestimmen, wird dieser Bias immer patientenrelevanter.

Hochwertige klinische Studien sind eine wichtige Voraussetzung für eine optimale Patientenversorgung. Ein niedriges Qualitätsniveau können wir uns nicht leisten. Als Fachgesellschaft sollten wir deshalb nicht müde werden, für Qualität und Transparenz einzutreten und die Wissenslücken in der Onkologie zu thematisieren, die im Interesse unserer Patienten vorrangig gefüllt werden müssen.

Ihr

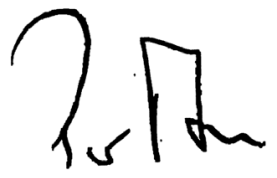

Ihr Peter Albers

Präsident der Deutschen Krebsgesellschaft 